\title{
Analysis on the influence of fuze warhead distance to the fuze warhead coordination
}

\author{
Shaoyong Jian ${ }^{1}$,Shi Ying * \\ ${ }^{1}$ Department of Mathematics and Computer Sciences, Xinyu University, Xinyu,338004,China \\ * Department of fundamental courses ,Wuhan Technology and Business University Wuhan, Hubei,430065,China
}

\begin{abstract}
For anti-aircraft missiles to intercept the small size of the object in a small miss distance condition cited war with the problem, analyze the warhead to Fuze distance interception prejudice to such objectives, through theory and simulation analysis, the distance is small miss distance to intercept small size of the target with the effect of the lead battle influence evident in the actual engineering design, we need to consider the impact of the distance to lead the war with the design of the proposed boundary distance constraints.
\end{abstract}

\section{Introduction}

Past air defense missile guidance deviation is bigger, in the design and analysis of fuze-warhead coordination, warhead and fuze is overlap, this assumption in the miss distance is reasonable, is largely due to the fuze trigger delay time of flight $\left(t_{f}\right)$ greater than the fragment $\left(t_{v 0}\right.$, flying fragment to a point of time) with the inherent delay fuze and $\left(\tau_{0}\right)$, so as to get the best fuze delay (r) is larger, the warhead and fuze distance $\left(D_{x 1}\right)$ in the direction of relative velocity $\left(V_{F}\right)\left(\Delta \tau=D_{X 1} / V_{F}\right)$ is the best time delay error caused by the delay of proportion is very small, and thus has little influence on fuze average starting position.With more and more small deviation guided anti-aircraft missiles, the presence of trigger delay fuze fragments smaller than the inherent delay of flight time and the circumstances, and if you do not consider the impact on the optimal delay will result in less than optimal delay 0 , average starting position relative to the fuze warhead fragments scattered region lagging behind, unable to achieve the desired effect with the lead battle, therefore, a small amount off target intercept targets need to consider the impact of the issue of war with the lead.

\section{2.issues analysis}

For a short-range interceptor weapon system, select the feature points (see Table 1) with the analysis cited war, warfare systems cited basic parameters are as follows: First Fragment velocity $2000 \mathrm{~m} / \mathrm{s}$, broken piece of the main scattering direction angle of $90^{\circ}$, inclination fuze antenna $50^{\circ}$, the inherent latency of $1.0 \mathrm{~ms}$, fuze warhead to a distance of $0.8 \mathrm{~m}$, the more typical trajectory without considering the lead to war under warhead fuze with effect from the condition shown in Figure 1.

Table 1 typical trajectory points

\begin{tabular}{|c|c|c|c|c|c|c|}
\hline Z & 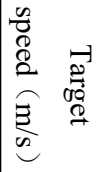 & 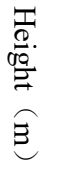 & 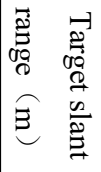 & 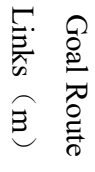 & 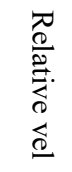 & 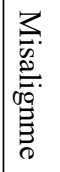 \\
\hline 1 & 300 & 500 & 3000 & 0 & 719 & 15 \\
\hline 2 & 300 & 500 & 3000 & 1450 & 710 & 17 \\
\hline
\end{tabular}

From the lead battle with the effects of simulation map view, without considering the distance fuze to warhead, fuze average starting position at $1 \mathrm{~m}$ off target amount condition lags Warhead dynamic scattering area, that broken pieces scattering area can not cover the target key points, features point lead with ineffective war, after analysis of the optimal delay models, that the main reason is the distance to the warhead fuze impact on the battle with the effects of lead. 


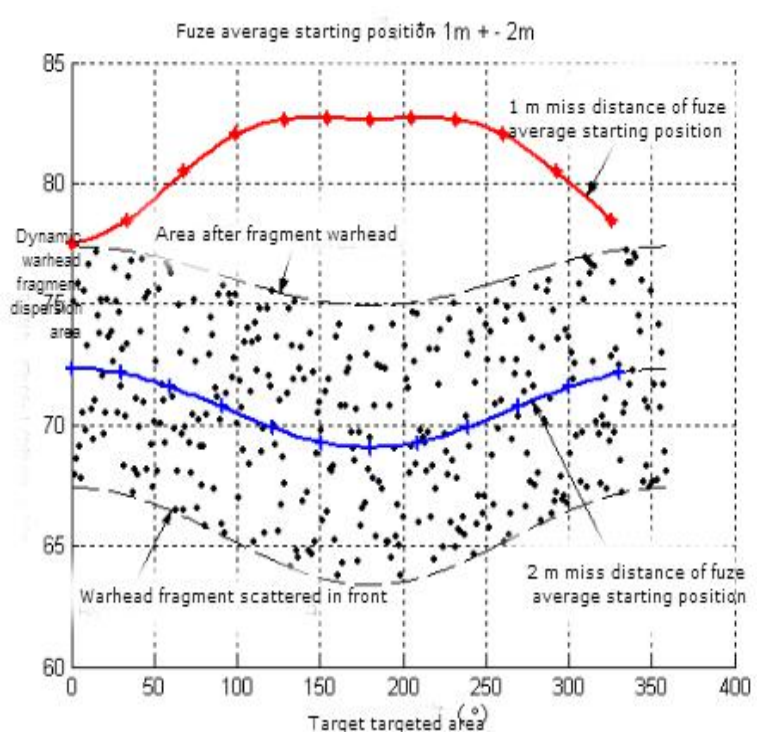

(1\#)

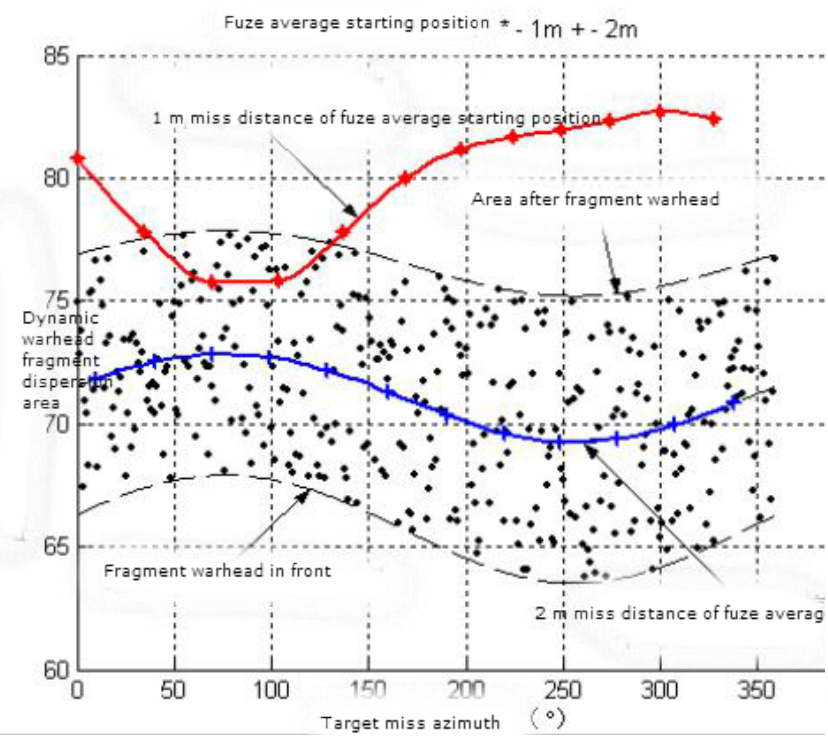

(2\#)

Figure 1 does not consider the conditions lead to the warhead a distance of 1 \# and 2 \# ballistic cited war with renderings

\subsection{Quantitative analysis of the impact of best}

\section{delay $D_{x 1}$}

In order to further analyze the effect of $D_{x 1}$ on the lead and the delay time delay, with the following delay from the simplified calculation model lead war starting, quantitative analysis of the issue, in the missile and the target is on or near the intersection opposite the intersec -tion (misalignment angle less than $15^{\circ}$ ) conditions, elastic mesh intersection model and delay formulas are as follows:

$$
\tau=t_{f}-t_{V 0}-\tau_{0}=\frac{\Delta L+\frac{\rho}{\tan \left(\Omega_{f}\right)}+D_{x 1}-\rho \tan (\delta)}{V_{r}}-\frac{\frac{\rho}{\cos (\delta)}}{V_{0}}-\tau_{0}
$$

Type: $\tau$ for Fuze optimal burst delay time; $\rho$ for the target miss distance; $\Omega_{f}$ for the tilt angle of antenna peak; $\Delta L$ for a target trigger point to the target key center distance $(\Delta L=0$ that target trigger point is the

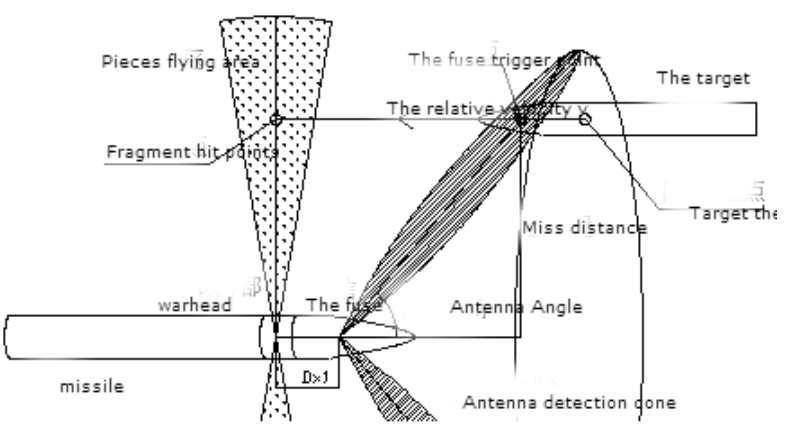

Figure 2 Missile project is on the intersection of computational geometry model best delay

target key points, $V_{r}$ for missile target relative velocity; $V_{0}$ as the initial velocity of the fragments $\delta$ fragment dispersion main direction angle of anteversion or retroversion angle (forward positive and backward tilt is negative)[1].

Assume that the target off target amount of change in the range of $0 \mathrm{~m} \sim 30 \mathrm{~m}$, fuze antenna peak inclination of $50^{\circ}, D_{x 1}$ is $0.8 \mathrm{~m}$, the relative speed of $800 \mathrm{~m} / \mathrm{s}$, early fragment velocity of $2000 \mathrm{~m} / \mathrm{s}$, broken piece of the main scattering direction is $90^{\circ}$, fuse inherent delay as $1.0 \mathrm{~ms}$, based on the above assumptions, analysis $\Delta \tau$ of the fuze optimal initiating delay time $\tau$ influence, under condition of different target volume $\Delta \tau$ of the best delay $\tau$ accounts for the proportion $(\Delta \tau / \tau)$ as shown in Figure 3 shows.

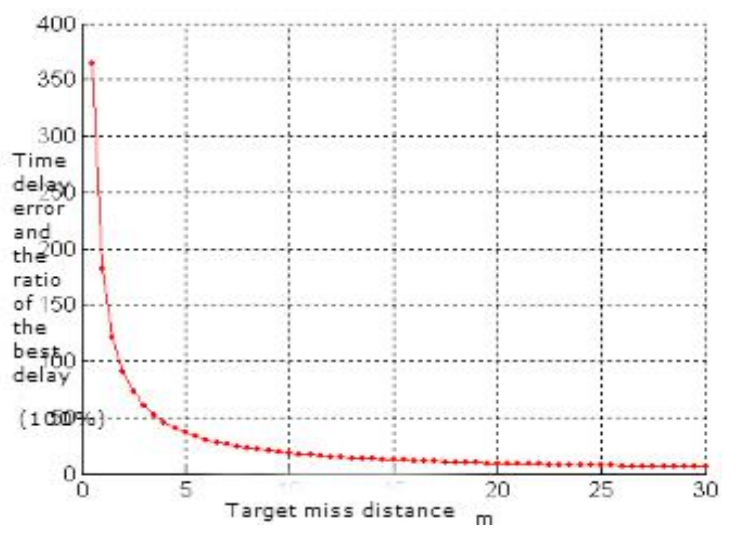

Figure 3 Delay error caused delays accounted for 
optimum ratio $(100 \%)$

From the map view, at $0.5 \mathrm{~m}, 1 \mathrm{~m}, 2 \mathrm{~m}$ and $3 \mathrm{~m}$ respectively at the proportion of $364 \%, 182 \%, 91 \%$ and $61 \%$, while in the $10 \mathrm{~m}, 20 \mathrm{~m}$ and $30 \mathrm{~m} \Delta \tau$ accounted for $\tau$ the ratio was $18 \%, 9 \%$ and $6 \%$, When the miss distance is smaller, $\Delta \tau$ to $\tau$ the proportion of larger, the fuze average starting position is greater, the greater the impact on the final fuze warhead coordinate on effect.

At the time of fuze warhead coordination analysis, in order to ensure the fuze average starting position not outside the fragment dynamic scattering section, theory, caused by $\Delta \tau$ the fuze average starting position change is less than $1 / 2$ the fragment dynamic scattering section width, namely:

$$
\Delta \tau \times V_{\text {rmax }} \leq \frac{\rho \varphi}{2}
$$

Where $\phi$ is the width of fragmentation dynamic scattering region.

\section{2 pairs $D_{x 1}$ boundary conditions constraint}

For formula 1, in the best delay is not less than zero conditions, in order to ensure fuze average starting position is not lagging behind the fragmentation dynamic scattering area at this time, when the miss distance tends to $0(\Delta L=0)$, the presence of the following formula :

$$
\lim _{\rho \rightarrow 0}\left[\frac{\frac{\rho}{\tan \left(\Omega_{f}\right)}+D_{X 1}+\Delta L}{V_{r}}-\frac{\rho}{V_{0} \sin (\theta)}-\tau_{0}\right]=\frac{D_{X 1}}{V_{r}}-\tau_{0} \geq 0
$$

Where by $D_{x 1}$ need to satisfy the following formula:

$$
D_{x 1} \geq V_{r \max } \times \tau_{0}
$$

Where $D_{\mathrm{x} 1}$ is the distance to warhead fuze, $V_{r \text { max }}$ is the largest bomb target relative veloci-ty, $\tau_{0}$ is the inherent delay fuse. Actual engineering design, you need to consider the trade-off relationship $D_{\mathrm{x} 1}, V_{r} \max$ and $\tau_{0}$ between reasonable design specifications, equipment layout after preliminary determination on the bomb, cited warfare systems need to focus on the inherent time delay fuse indicators, for example, the maximum relative speed $800 \mathrm{~m} / \mathrm{s}$, the distance to the warhead fuze is under $0.8 \mathrm{~m}$ condition, the inherent delay fuse not greater than $1 \mathrm{~m} / \mathrm{s}$.

In $D_{x 1}$ can not satisfy Formula 3 missile to intercept the target's lead war with Figure 1, in a small miss distance, the fuze average starting position outside the fragmentation dynamic scattering area range, lead to the removal of the target amount warhead can not hit the target key point.

\section{Model Improvement}

Best Start Delay Fuze engineering calculation model, the distance to the warhead fuze fuze trigger delay mainly affects the calculation has no impact on fragments flying time, the inherent delay and off target azimuth correction time, consider the following fuze to fight the influence of the distance portion of the original model to improve the trigger delay.

\subsection{Coordinate system definition}

This paper relates to the coordinate system of the ground, elastomers and relative velocity coordinates, the concept of indirect references to other coordinate systems, see the relevant single shot kill probability models to calculate the model file [2].

Ground we coordinate system: ${ }_{o x} d^{y} d_{d}^{z}$ ground coordina -te system represented by qwer, which is defined as: the origin 0 is located in the missile launch point, ${ }_{o z}$ instantaneous axis of missile target flight speed parallel projection on the ground, and taking the opposite direction is positive, ${ }^{o y}{ }_{d}$ axis vertical upward is positive, $o_{d}$ axis and $o_{d}$ and $o_{d}$ right-handed coordinate system constituted.

Body coordinates: With $o x_{1} y_{1} z_{1}$ indicates the origin of the coordinate system projectile fuze is located in the center of missiles along the longitudinal axis of the shaft $o x_{1}$ forward, $o y_{1}$ to take in the missile body axis symmetry plane, constitute $o z_{1}$ right-handed coordinate system.

Relative velocity coordinates: With oxyz indicates. o take the origin of coordinates in the target centroid, oz axis in the opposite direction to take missiles opposite direction of the target velocity vector $V_{r}$ is positive, as oy axis perpendicular to the axis oz, ox axis constituted by right-handed rule.

From the body coordinate system to the coordinate 
system relative velocity transformation matrix:

$A_{1 x}=A_{d x} \times A_{1 d}=M_{x}\left[\varepsilon_{r}\right] M_{y}\left[\beta_{r}-90\right] \times M_{y}[-\psi] M_{z}[-\nu] M_{x}[-\gamma]$

Where, $A_{d x}$ is ground coordinate system to convert the coordinate system relative velocity matrix, $\varepsilon_{r}$ is the relative trajectory angle, $\beta_{r}$ is the relative trajectory yaw angle; $A_{1 d}$ is body coordinates to the ground coordinate system transformation matrix, $\psi, v$ and $\gamma$ respectively projectile yaw angle, pitch angle and projectile missile body roll angle.

\section{2 trigger delay calculation model}

In the relative velocity coordinates, to establish the trigger delay calculation geometry shown in Figure 4, the figure, $\mathrm{O}$ points for the target organism center, $\mathrm{A}$ point relative velocity coordinate the play off target coordinates $(\rho \cos \theta, \rho \sin \theta, 0)$, B point for the target trigger points relative velocity coordinate system position coordinates $\left(x_{i}, y_{i}, z_{i}\right), \mathrm{N}$ point fuze center position coordinates relative velocity, the distance off the target for $V_{r} \times t_{i}, t_{i}$ to trigger time, $\mathrm{M}$ point for the location warhead fuze center relative to the center of the relative speed of the coordinate system, the position of its center is relatively fuze $\left(D_{r x 1} 、 D_{r y 1}, D_{r z 1}\right)$, which is calculated, see equation (14).

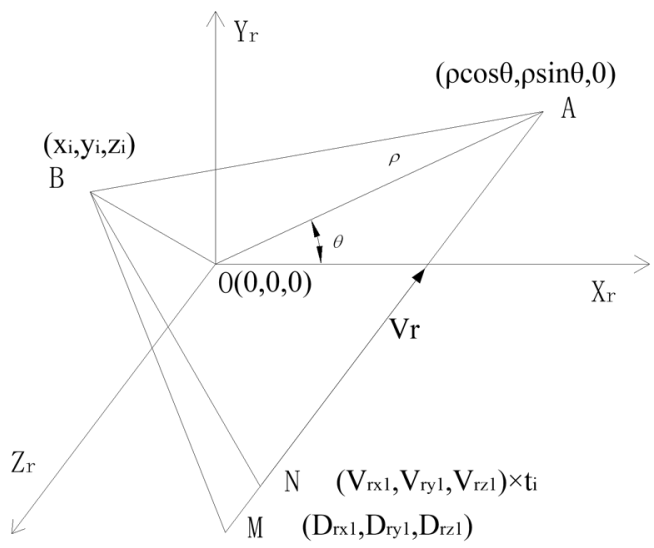

Figure 4 Trigger Point Delay computational geometry model

In Figure 4, the coordinate system relative velocity, $A B$ is represented as follows [3]:

$O A=(\rho \cos \theta, \rho \sin \theta, 0)$
$O B=\left(x_{i}, y_{i}, z_{i}\right)$

$A B=O B-O A=\left[\begin{array}{c}x_{i}-\rho \cos \theta \\ y_{i}-\rho \sin \theta \\ z_{i}\end{array}\right]$

When playing the system, consider the warhead center position vector with respect to the center of the fuse $\overrightarrow{M N}$, and $\overrightarrow{A B}$ converted from the relative velocity vector coordinates to the missile body coordinates, the position of the trigger point in playing the system $i$ have the following relationship:

$$
\begin{aligned}
& M N=\left(D_{X 1}, D_{y 1}, D_{Z 1}\right) \quad(9) \\
& N A=\left(V_{r X 1}, V_{r y 1}, V_{r Z 1}\right) \times t_{i} \quad(10) \\
& M A=M N+N A=\left[\begin{array}{c}
D_{X 1} \\
D_{y 1} \\
D_{Z 1}
\end{array}\right]+\left[\begin{array}{c}
V_{r X 1} \\
V_{r y 1} \\
V_{r z 1}
\end{array}\right] \times t_{i} \quad(11) \\
& \overrightarrow{M B}=\overrightarrow{M A}+\overrightarrow{A B}=\left[\begin{array}{l}
x_{1 i} \\
y_{1 i} \\
z_{1 i}
\end{array}\right]=A_{x 1} \times\left[\begin{array}{c}
x_{i}-\rho \cos \theta \\
y_{i}-\rho \sin \theta \\
z_{i}
\end{array}\right]+\left[\begin{array}{l}
D_{x 1} \\
D_{y 1} \\
D_{z 1}
\end{array}\right]+\left[\begin{array}{l}
V_{r x 1} \\
V_{r y 1} \\
V_{r z 1}
\end{array}\right] \times t_{i}
\end{aligned}
$$

In the formula, $D_{x 1}, D_{y 1}$ and $D_{z 1}$ warhead missile system in the center position with respect to the fuse center.

According to the actual engineering computing needs, location of the point system will trigger bomb formula 12 is converted to the following formula:

$$
\begin{aligned}
& {\left[\begin{array}{c}
x_{1 i} \\
y_{1 i} \\
z_{1 i}
\end{array}\right]=A_{x 1} \times\left[\begin{array}{c}
x_{i}-\rho \cos \theta+D_{r x 1} \\
y_{i}-\rho \sin \theta+D_{r y 1} \\
z_{i}+D_{r z 1}
\end{array}\right]+\left[\begin{array}{c}
V_{r x 1} \\
V_{r y 1} \\
V_{r z 1}
\end{array}\right] \times t_{i}} \\
& {\left[\begin{array}{c}
D_{r x 1} \\
D_{r y 1} \\
D_{r z 1}
\end{array}\right]=A_{1 x} \times\left[\begin{array}{c}
D_{x 1} \\
D_{y 1} \\
D_{z 1}
\end{array}\right]}
\end{aligned}
$$

Equation (13) is considered relatively fuze warhead fuze position after the trigger delay calculation model, using ground test trigger line fuse data, combined with the above formula iterative solver get fuze trigger delay, and then calculate the best start delay fuse, be analysis of lead battle with effect[4]. 


\section{Simulation Analysis}

Through the delay calculation model improvement, re-lead war with the simulation analysis, Table 1, the two feature points lead battle simulation with the effect shown in Figure 5.

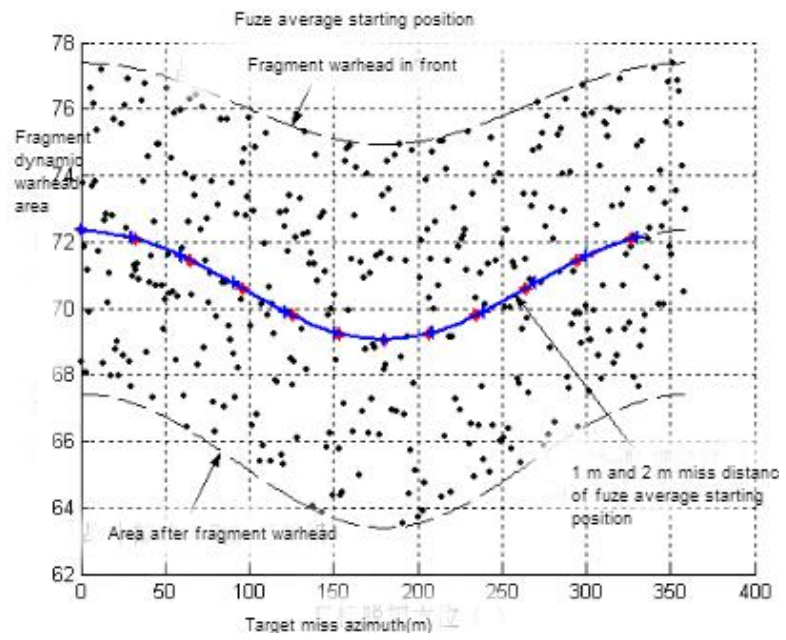

(3\#)

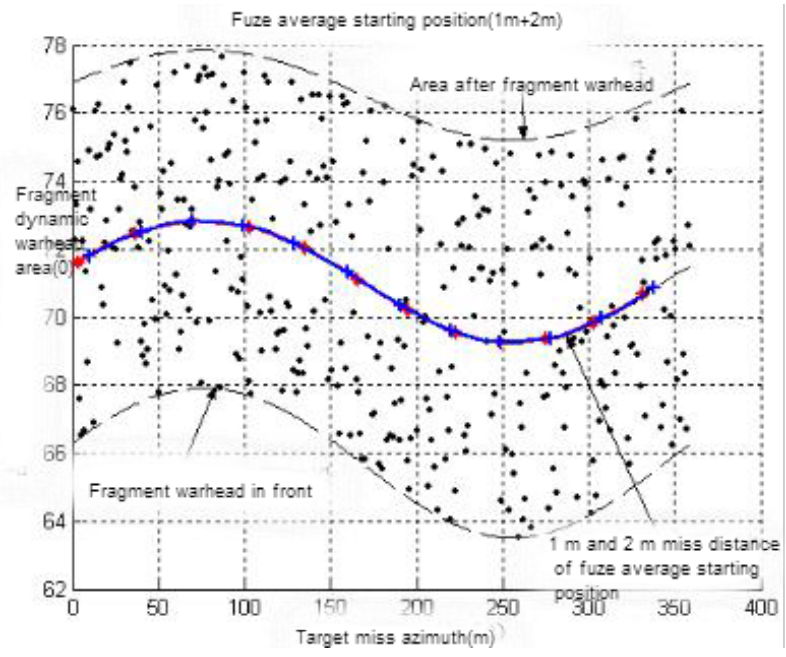

(4\#)

Figure 5 The improved design 3\# and 4\# ballistic cited war with effect simulation map

From Figure 5, the consideration of the impact fuze distance to warhead, improved trigger delay calculation model, citing the war on the orientation of each off-target with good effect, trigger delay calculation model improvements achieved to improve the effective -ness of combining the purpose of the lead battle, to meet the target of high-precision missile defense requirements[5].

\section{Conclusion}

With the presence of small off-target for the amount of lead war to intercept targets poor problem, through theoretical analysis and model to improve that when a small amount off target interception target, you need to consider the impact of distance warhead fuze to war with the effects of lead, and the distance and delays inherent in the relationship between the fuze given constraint equation, cited improved model with war simulation results show that the missile intercept targets with a good primer war.

\section{References}

1 Zhihong Zhang air defense missile and warhead fuze andwarhead design with efficiency Beijing: Astronautics Publishing House .(1994)

2 Tongwen Liang antiaircraft missile fuze design and simulation technology. Beijing: Astronautics Publishing House .(1994)

3 Bozhen Cao winged missile warhead and fuze Beijing: Astronautics Publishing House. (1995)

4 Patrick Girard, Nicola Nicolici, Xiaoqing Wen Power-Aware Testing and Test Strategies for Low Power Devices, $[\mathrm{M}]$ Springer Science \& Business Media, Technology \& Engineering, pp.297-300, 2010 .

$5 \mathrm{X}$. Lin and Y. Huang, "Scan shift power reduction by freezing power sensitive scan cells, " Journal of Electronic Testing, vol. 24, no. 4, pp. 327 -334, 2008. 